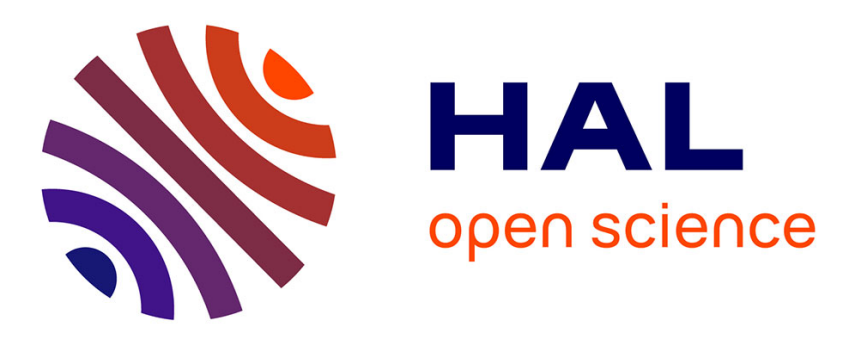

\title{
Practical Implementation of Integer Forcing Linear Receivers in MIMO Channels
}

\author{
Asma Mejri, Ghaya Rekaya-Ben Othman
}

\section{To cite this version:}

Asma Mejri, Ghaya Rekaya-Ben Othman. Practical Implementation of Integer Forcing Linear Receivers in MIMO Channels. Vehicular Technology Conference Fall, Sep 2013, Las Vegas, United States. pp.1-5. hal-00874966

\section{HAL Id: hal-00874966 https://hal.science/hal-00874966}

Submitted on 19 Oct 2013

HAL is a multi-disciplinary open access archive for the deposit and dissemination of scientific research documents, whether they are published or not. The documents may come from teaching and research institutions in France or abroad, or from public or private research centers.
L'archive ouverte pluridisciplinaire HAL, est destinée au dépôt et à la diffusion de documents scientifiques de niveau recherche, publiés ou non, émanant des établissements d'enseignement et de recherche français ou étrangers, des laboratoires publics ou privés. 


\title{
Practical Implementation of Integer Forcing Linear Receivers in MIMO Channels
}

\author{
Asma Mejri and Ghaya Rekaya-Ben Othman \\ Telecom ParisTech, 46 Rue Barrault, 75013 Paris, France, amejri,rekaya@telecom-paristech.fr
}

\begin{abstract}
Integer Forcing (IF) architecture has been recently proposed to design linear receivers in MIMO systems. Research works show the promise of this architecture from a capacity achieving perspective. However, it is not totally understood how to select IF coefficient matrix and if the promised theoretical gain of the resulting receivers is attainable in practical settings. We try in this work to fill the gap between theory and practice: we propose algorithms to select optimal IF receiver parameters that lead to the maximization of the total achievable rate. We propose an implementation of an IF-based MIMO system considering a practical scenario where lattice codes are used. Experimental studies are carried out to evaluate the error rate performance of the proposed algorithms and compare them to traditional linear receivers. Our proposed implementation shows that the theoretical potential of the IF receivers is achievable even with finite-length lattice codes.
\end{abstract}

Index Terms-MIMO systems, linear receivers, lattice reduction, lattice coding, Integer Forcing Linear receivers

\section{INTRODUCTION}

$\mathrm{W}$ consider transmission over a Multiple Input-Multiple Output (MIMO) channel without space-time coding and investigate a new architecture of linear receivers termed Integer Forcing Linear Receivers. Recently proposed by Jiening et al. in [1],[2],[3] and [4], this architecture is based on the use of structured codes for channel coding. Upon utilizing the same code separately at the transmit antennas, achievable rates are proved to go highly beyond those attainable using traditional linear receivers. The idea is that, instead of creating independent interference-free data flows at the different receive antennas and decode each codeword separately, as is the case of the Zero-Forcing (ZF) and Minimum Mean Square (MMSE) detectors, both interference and code's linearity are exploited to decode integer linear combinations of original codewords. Upon decoding a full rank set of codewords' combinations according to an integer full rank coefficient matrix, original messages can be recovered by a simple matrix inversion.

Works in [1] and [3] show the promise of the new IF architecture from a capacity achieving perspective and propose a design criteria for the optimal integer coefficient matrix. What is missing is to develop methods to solve the integer optimization problem and to understand the error performance of the IF receivers in practical scenarios. In this work we address these remaining issues:

- We propose algorithms to select optimal IF coefficient matrix such that the total achievable rate is maximized.

- We propose a practical implementation of IF receiversbased MIMO systems where a low-complexity lattice coding scheme is considered, and evaluate the error rate performance. Traditional linear receivers, namely the ZF and the MMSE as well as Lattice Reduction-aided linear receivers under the LLL reduction are included for comparisons. Our experimental studies show that the potential of the new IF design over standard linear receivers is attainable in practical settings even using finite-length lattice codes.

The remaining flow of this work is organized as follows: in a second section we describe the system model and set up the optimization problem. In sections III and IV we respectively detail the proposed algorithms and practical implementation of IF receivers. Performance evaluation is the subject of section $\mathrm{V}$ followed by a concluding section.

\section{SYSTEM MODEL AND PROBLEM SET UP}

Two key requirements characterize the IF design: a same lattice code is used for channel coding and encoded data streams are independent at the different transmit antennas.

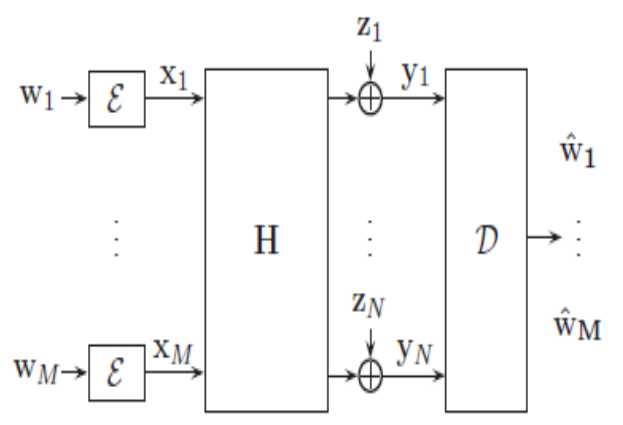

Fig. 1. MIMO system with linear independent encoding.

According to these requirements and following the original scheme of [3], we consider a MIMO channel composed of $M$ transmit and $N$ receive antennas $(M \leq N) \cdot m^{t h}$ transmit antenna delivers a length $k$ message $\mathbf{w}_{m}$, drawn i.i.d from a finite field $\mathbb{F}_{p}^{k}$ and encodes it separately onto an $n$-dimensional lattice codeword $\mathbf{x}_{m}$ to form the channel input as illustrated in Fig.1. Transmit antennas are equipped with a same lattice encoder $\mathcal{E}: \mathbb{F}_{p}^{k} \rightarrow \mathbb{R}^{n}$ that maps the finite field messages to codewords. Encoded vectors satisfy a symmetric power constraint given by $\frac{1}{n}\left\|\mathbf{x}_{m}\right\|^{2} \leq P$. For ease of presentation, real channel model is considered. Received signal can then be 
expressed in the form:

$$
\mathbf{Y}_{N \times n}=\mathbf{H}_{N \times M} \mathbf{X}_{M \times n}+\mathbf{Z}_{N \times n}
$$

where $\mathbf{H}$ denotes the real valued fading channel of entries generated i.i.d. according to a zero-mean unit variance normal distribution. $\mathbf{Z}$ models a zero-mean additive white Gaussian noise of variance $\sigma^{2}$ and $\mathbf{X}$ is the channel input of rows corresponding to the transmitted codewords $\mathbf{x}_{1}, \mathbf{x}_{2}, \ldots, \mathbf{x}_{M}$. We assume a perfect Channel State Information at the receiver (only) and a flat fading channel where $\mathbf{H}$ remains constant over the transmission of the whole codewords. We denote the Signal to Noise Ratio (SNR) by $\rho=\frac{P}{\sigma^{2}}$.

\section{A. Related work}

Traditional decoders for MIMO systems aim to decode original codewords individually. Maximum Likelihood (ML) decoder offers optimal performance at the expense of high complexity. Motivated by their low computation power consumption, alternative linear receivers such as the ZF and the MMSE are deployed in wireless systems limited by processing capabilities. In such detectors, the receiver attempts to create interference-free data flows at each receive antenna by a projection of the received signal with a preprocessing matrix $\mathbf{B} \in \mathbb{R}^{M \times N}$. This matrix for the $\mathrm{ZF}$ and the MMSE is:

$$
\mathbf{B}_{\mathrm{ZF}}=\left(\mathbf{H}^{t} \mathbf{H}\right)^{-1} \mathbf{H}^{t}, \mathbf{B}_{\mathrm{MMSE}}=\mathbf{H}^{t}\left(\mathbf{H H}^{t}+\frac{1}{\rho} \mathbf{I}\right)^{-1}
$$

Although simple, these receivers have poor performance in terms of diversity-multiplexing tradeoff. Their corresponding achievable rate for the $m^{\text {th }}$ data stream is given by [2],

$$
R_{m, \operatorname{Lin}}=\frac{1}{2} \log \left(1+\frac{\rho\left\|\mathbf{b}_{m}^{t} \mathbf{H}\right\|^{2}}{\left\|\mathbf{b}_{m}^{t}\right\|^{2}+\rho \sum_{i \neq m}\left\|\mathbf{b}_{i}^{t} \mathbf{H}\right\|^{2}}\right)
$$

where $\mathbf{b}_{i}^{t}$ represents the $i^{\text {th }}$ row of the matrix $\mathbf{B}$.

In order to enhance the performance of linear receivers, Lattice Reduction (LR) techniques are used as a preprocessing stage to improve the orthogonality of the channel matrix. A reduction of $\mathbf{H}$ gives a near orthogonal matrix $\mathbf{H}_{\mathrm{r}}$ related to the former by $\mathbf{H}_{\mathrm{r}}=\mathbf{H T}$, with $\mathbf{T}$ a unimodular matrix. Preprocessing matrices of LR-aided linear receivers are the following:

$$
\begin{aligned}
& \mathbf{B}_{\mathrm{LR}-\mathrm{ZF}}=\left(\mathbf{H}_{\mathrm{r}}^{t} \mathbf{H}_{\mathrm{r}}\right)^{-1} \mathbf{H}_{\mathrm{r}}^{t} \\
& \mathbf{B}_{\mathrm{LR}-\mathrm{MMSE}}=\left(\mathbf{T}^{t} \mathbf{T}\right)^{-1} \mathbf{H}_{r}^{t}\left(\mathbf{H}_{r}\left(\mathbf{T}^{t} \mathbf{T}\right)^{-1} \mathbf{H}_{r}^{t}+\frac{1}{\rho} \mathbf{I}\right)^{-1}
\end{aligned}
$$

\section{B. Integer-Forcing Linear Receivers}

In both linear receivers and LR-aided linear receivers, the interference provided by the channel is cancelled and the linearity of the underlying code is not exploited. The main motivation to the design of the Integer Forcing linear receivers is to take advantage of the code's structure to make the interference a beneficial characteristic of the wireless medium: receive antennas will decode, from the channel output, integer linear combinations of all original codewords including both desired and interfering signals according to an integer full rank coefficient matrix. Thanks to the linear structure of the used code, these linear combinations are also codewords.

Due to the real nature of the fading channel, observed combinations at different antennas are real and do not correspond to lattice codewords. An additional processing is then required. The idea is to quantize the channel matrix $\mathbf{H}$ with a full rank integer matrix $\mathbf{A} \in \mathbb{Z}^{M \times M}$. This approximation is made by preprocessing the channel output by some matrix B. Mathematically, it corresponds to get $\tilde{\mathbf{Y}}$ such that:

$$
\tilde{\mathbf{Y}}=\mathbf{B Y}=\mathbf{A X}+(\mathbf{B H}-\mathbf{A}) \mathbf{X}+\mathbf{B Z}
$$

Scaled signal at the $m^{t h}$ antenna can be written as,

$$
\tilde{\mathbf{y}}_{m}=\underbrace{\sum_{j=1}^{M} a_{m j} \mathbf{x}_{j}}_{\lambda_{m}}+\sum_{j=1}^{M}\left(b_{m j} H_{m j}-a_{m j}\right) \mathbf{x}_{j}+\sum_{j=1}^{M} b_{m j} \mathbf{z}_{j}
$$

where $\mathbf{a}_{m}^{t}=\left[a_{m 1}, \ldots, a_{m M}\right]$ and $\mathbf{b}_{m}^{t}=\left[b_{m 1}, \ldots, b_{m M}\right]$ are the $m^{t h}$ row vectors of $\mathbf{A}$ and $\mathbf{B}$ respectively. The $m^{t h}$ receive antenna is equipped with a separate decoder $\mathcal{D}_{m}$ that attempts to decode from $\tilde{\mathbf{y}}_{m}$ the integer combination $\lambda_{m}$ of coefficient vector $\mathbf{a}_{m}$. Then, $\Lambda=\left[\lambda_{1}, \lambda_{2}, \ldots, \lambda_{M}\right]^{t}=\mathbf{A X}$ is constructed to recover original codewords by a simple inversion of the matrix A. Finite field messages are afterwards estimated by mapping the decoded codewords back to the finite field. Block diagram of IF receivers is illustrated in Fig.2.

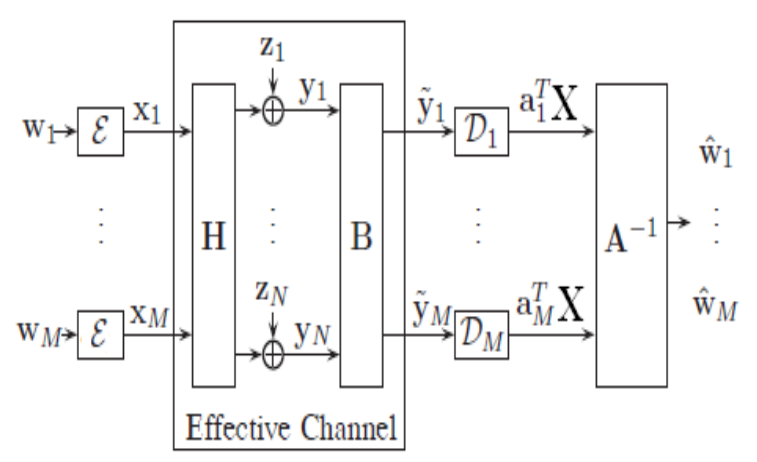

Fig. 2. Block diagram of Integer Forcing Linear Receivers in MIMO systems.

Remark: Linear receivers as well as LR-aided linear receivers can be seen as particular cases of the IF design. Considering their above mentioned preprocessing matrices, their corresponding equivalent channel matrices are respectively the identity matrix and the unimodular matrix $\mathbf{T}$. Through an example, authors in [3] show the suboptimality of restricting the equivalent channel matrix to be unimodular. As a proof of concept, we implement in this work a low-complexity lattice reduction technique which is the LLL reduction [5] and analyze its performance compared to the Integer Forcing receivers.

In order to build IF receivers and find preprocessing matrix $\mathbf{B}$ and coefficient matrix $\mathbf{A}$, authors in [2] propose a design criterion based on the maximization of the total sum rate. They 
show that the achievable computation rate for the $m^{\text {th }}$ data stream, for a fixed coefficients vector $\mathbf{a}_{m}$, is:

$$
R_{m, \mathrm{IF}}=\frac{1}{2} \log ^{+}\left(\frac{\rho}{\left\|\mathbf{b}_{m}^{t}\right\|^{2}+\rho\left\|\mathbf{b}_{m}^{t} \mathbf{H}-\mathbf{a}_{m}^{t}\right\|^{2}}\right)
$$

Then their design criterion corresponds to the maximization of the minimum computation rate achievable over all receive antennas. Based on this, optimal preprocessing vector $\mathbf{b}_{m}$ for a fixed coefficient vector $\mathbf{a}_{m}$, was found in [2] as:

$$
\mathbf{b}_{m, \mathrm{opt}}=\mathbf{a}_{m} \mathbf{H}^{t}\left(\mathbf{H} \mathbf{H}^{t}+\frac{1}{\rho} \mathbf{I}\right)^{-1}
$$

Then, by replacing in the expression of the achievable rate the preprocessing vector by its optimal value, the resulting achievable rate at receiver $m$ becomes [3]:

$$
R_{m, \text { IF }}\left(\mathbf{a}_{m}\right)=-\frac{1}{2} \log \left(\mathbf{a}_{m}^{t} \mathbf{V D V}^{t} \mathbf{a}_{m}\right)
$$

where $\mathbf{V} \in \mathbb{R}^{N \times N}$ is the unitary matrix whose columns are the right singular vectors of $\mathbf{H}$ and $\mathbf{D}$ is a diagonal matrix with elements

$$
D_{i i}= \begin{cases}\frac{1}{1+\rho \sigma_{i}^{2}} & \text { if } i \leq \operatorname{rank}(\mathbf{H}) \\ 1 & \text { if } i>\operatorname{rank}(\mathbf{H})\end{cases}
$$

where $\sigma_{i}$ is the $i^{t h}$ singular value of the channel matrix $\mathbf{H}$. Optimal coefficient vectors are then selected such that the minimum achievable rate over all receive antennas is maximized under the full rank constraint. They are solution of the following integer optimization problem:

$$
\mathbf{a}_{m, \mathrm{opt}}=\underset{\substack{|\mathbf{A}| \neq 0 / \\\left\|\mathbf{a}_{m}\right\|^{2} \leq 1+\rho \sigma_{\text {max }}^{2}}}{\operatorname{argmax}} \underset{m=1, \ldots, M}{\min }\left\{\mathbf{a}_{m}^{t} \mathbf{G} \mathbf{a}_{m}\right\}
$$

where $\mathbf{G}=\mathbf{V D V}^{t}$.

So far, this optimization problem was only proposed and no methods to solve it have been investigated. In the following sections, we will first propose practical algorithms that allow to find optimal coefficient vectors such that the total sum rate is maximized, then we propose a practical implementation using a low-complexity lattice coding scheme. We address in a last section performance evaluation of the proposed algorithms.

\section{PRoposed ALGORITHMS FOR OPTIMAL COEFFICIENT VECTORS SELECTION}

Let $Q(\mathbf{a})=\mathbf{a}^{t} \mathbf{G a}$. Given that $\mathbf{G}$ is symmetric definite positive, it has a Cholesky decomposition $\mathbf{G}=\mathbf{R}^{t} \mathbf{R}$ and we can write $Q(\mathbf{a})=\mathbf{a}^{t} \mathbf{R}^{t} \mathbf{R} \mathbf{a}=\|\mathbf{R} \mathbf{a}\|^{2}$. Minimization of the quadratic form $Q$ remains then to a Shortest Vector Problem and solving optimization problem (9) remains to search the Shortest Linearly Independent Vectors of the lattice $\Lambda_{\mathbf{G}}$ of Gram matrix $\mathbf{G}$ that satisfy $\left\|\mathbf{a}_{m}\right\|^{2} \leq 1+\rho \sigma_{\max }^{2}$.

In literature, lattice decoding and reduction methods are used to solve the Shortest Vector Problem. In our method we adopt the Fincke-Pohst algorithm [6] and adapt it to meet the requirement of the integer optimization problem. Our search method is based on two phases: i) Enumeration of all non-zero integer vectors $\mathbf{t}$ such that $\mathbf{t}^{t} \mathbf{G t}$ is minimized.

ii) Selection of the best linearly independent vectors.

We detail in the following paragraphs these two phases separately.

\section{A. Enumeration of integer vectors}

The obvious way to accomplish this phase is to perform an exhaustive search over all non-zero integer vectors in the set $\mathcal{A}=\left\{\mathbf{t} \in \mathbb{Z}^{M},\|\mathbf{t}\|^{2} \leq 1+\sigma_{\max }^{2} \rho\right\}$ that minimize the quadratic form $Q$. However this method has a complexity that increases as a function of the SNR. In our proposed approach, we reduce the search space set to a sphere of radius $C$ and solve for integer vectors such that:

$$
Q(\mathbf{t})=\mathbf{t}^{t} \mathbf{G} \mathbf{t} \leq C
$$

By performing the Cholesky decomposition of $\mathbf{G}$, we can write (10) as:

$$
\sum_{i=1}^{M}\left(R_{i i} t_{i}+\sum_{j=i+1}^{M} R_{i j} t_{j}\right)^{2} \leq C
$$

where $\mathbf{t}=\left[t_{1}, t_{2}, \ldots, t_{M}\right]^{t}$. Or equivalently as

$$
\sum_{i=1}^{M} q_{i j}\left(t_{i}+\sum_{j=i+1}^{M} q_{i j} t_{i}\right)^{2} \leq C
$$

with $q_{i i}=R_{i i}^{2}, i=1, \ldots, M, q_{i j}=\frac{R_{i j}}{R_{i i}}, j=i+1, \ldots, M$ After carrying out computations for $i=M, \ldots, 1$, we obtain the following bounds for each possible value of $t_{i}$ :

$$
\begin{gathered}
\left\lceil-\sqrt{\frac{C}{q_{M M}}}\right\rceil \leq t_{i} \leq\left\lfloor\sqrt{\frac{C}{q_{M M}}}\right\rfloor \\
\left\lceil-\sqrt{\frac{T_{i}}{q_{i i}}}-U_{i}\right\rceil \leq t_{i} \leq\left\lfloor\sqrt{\frac{T_{i}}{q_{i i}}}-U_{i}\right\rfloor, i=M-1, \ldots, 1
\end{gathered}
$$

where

$$
\begin{aligned}
U_{i} & =\sum_{j=i+1}^{M} q_{i j} t_{j} \\
T_{i} & =T_{i+1}-q_{i+1, i+1}\left(t_{i+1}+\sum_{j=i+1}^{M} q_{i+1, j} t_{j}\right)^{2}
\end{aligned}
$$

Taking into account these bounds, we start with searching the $M^{\text {th }}$ component of the desired vector $\mathbf{t}$ then elements $t_{M-1}, \ldots, t_{1}$ are found according to the following algorithm: Input: Gram matrix $\mathbf{G}$ and a positive constant $C$.

Output: The set $\Omega=\left\{\mathbf{t} \in \mathcal{A} \backslash \mathbf{0}, \mathbf{t}^{t} \mathbf{G t} \leq C\right\}$ of cardinal $K$ solution of (10)

i) Perform Cholesky decomposition of $\mathbf{G}=\mathbf{R}^{t} \mathbf{R}$, set $q_{i i}=$ $R_{i i}^{2}, i=1, \ldots, M$ and $q_{i j}=\frac{R_{i j}}{R_{i i}}, j=i+1, \ldots, M$.

ii) (Initialization) Set $i=M, T_{i}=C, S_{i}=0$. 
iii) (Compute bounds for $t_{i}$ ) Set $Z=\sqrt{\frac{T_{i}}{q_{i i}}}, U B\left(t_{i}\right)=\lfloor Z-$ $\left.S_{i}\right\rfloor, L B\left(t_{i}\right)=\left\lceil-Z-S_{i}\right\rceil$ and set $t_{i}=L B\left(t_{i}\right)-1$.

iv) (Increase $t_{i}$ ) Set $t_{i}=t_{i}+1$. For $t_{i} \leq U B\left(t_{i}\right)$ go to vi), else go to $\mathrm{v}$ ).

v) (Increase i) Set $i=i+1$ and go to iv)

vi) (Decrease $i$ ) For $i=1$ go to vii), else set $i=i-1, S_{i}=$ $\sum_{j=i+1}^{M} q_{i j} t_{j}, T_{i}=T_{i+1}-q_{i+1, i+1}\left(t_{i+1}+S_{i+1}\right)^{2}$ and go to step iii).

vii) (Solution found) For $\mathbf{t}=\mathbf{0}$ terminate, else set $Q(\mathbf{t})=$ $C-T_{1}+q_{11}\left(t_{1}+S_{1}\right)^{2}$ and go to step iv).

\section{B. Selection of optimal coefficient vectors}

Once the set of integer vectors $\Omega$ is found, we select the best linearly independent vectors to form the desire full rank matrix A. Our algorithm for optimal vectors selection is the following:

Input: The set $\Omega$.

Output: Shortest Linearly Independent Integer vectors $\overline{\mathbf{a}_{m, \mathrm{opt}}}, m=1, \ldots, M$

i) Order the set $\Omega$ into $\Omega_{\text {ord }}$ based on the lengths of the vectors $\mathbf{t}_{1}, \ldots, \mathbf{t}_{K}$ such that $\left\|\mathbf{R t}_{1}\right\|^{2} \leq \ldots \leq\left\|\mathbf{R t}_{K}\right\|^{2}$.

ii) Construct from $\Omega_{\text {ord }}$ the set $\mathcal{S}$ of $M$ lattice points $\mathbf{R t}_{1}, \ldots, \mathbf{R t}_{M}$ such that $\mathbf{t}_{1}, \ldots, \mathbf{t}_{M}$ are linearly independent.

iii) Adjust $C$ to guarantee that $|\mathcal{S}| \geq M$ (repeat the search of the set $\Omega$ ).

iv) Select the vectors $\mathbf{a}_{m, \mathrm{opt}}=\mathbf{t}_{m}, m=1, \ldots, M$.

\section{PRACTICAL IMPLEMENTATION OF IF-BASED MIMO SYSTEM}

In this section we describe the coding and decoding parts for IF-based MIMO systems. In a first subsection we will give some insights on the channel codes considered in the original scheme of [2] and we describe the code we use in our implementation. In a second subsection the processing steps at the receiver are described.

\section{A. Transmitter's architecture based on lattice coding}

Integer Forcing linear receivers use in their essence linear Capacity-achieving codes termed Nested Lattice Codes. Originally proposed by Erez and Zamir in [7], these codes are proved to approach the AWGN channel's capacity. They have been used later on in the framework of the Compute-andForward relaying strategy in [8]. In general, a lattice $\Lambda_{\mathrm{C}}$ is said to be nested in the lattice $\Lambda_{\mathrm{F}}$ if $\Lambda_{\mathrm{C}} \subset \Lambda_{\mathrm{F}} . \Lambda_{\mathrm{C}}$ and $\Lambda_{\mathrm{F}}$ are termed the Coarse and the Fine Lattice respectively. The Fine lattice represent the coding lattice from which are carved the codewords and the coarse lattice is the shaping lattice that ensures that the power constraint $P$ at the transmitter is satisfied. The set of all points of the Fine lattice that fall within the fundamental Voronoi Region $\mathcal{V}$ of the Coarse lattice constructs the nested lattice code $\mathcal{C}=\left\{\mathcal{V} \cap \Lambda_{\mathrm{F}}\right\}$ [9].

In the underlying MIMO systems, finite field messages $\mathbf{w}_{i}$ at transmit antennas are separately mapped to codewords $\mathbf{x}_{i}$ from the same nested lattice code $\mathcal{C}$ using a bijective mapping $\Phi: \mathbb{F}_{p}^{k} \rightarrow \mathcal{C}$ such that $\Phi\left(\mathbf{w}_{i}\right)=\mathbf{x}_{i}, i=1, \ldots, M$.
In practice, nested lattice codes can be designed based on linear codes or LDPC codes over finite fields. Let $C$ be a linear code over $\mathbb{Z}_{p}^{k}$ ( $p$ prime) with generator matrix $\mathbf{L}$. The coarse lattice can just be a scaled version of the integers by the field's size $p, \Lambda_{\mathrm{C}}=p \mathbb{Z}^{n}$ and the Fine lattice is generated by applying the Construction A to the linear code $C$. It consists of the following steps:

1) Construction, from the linear code $C$, of the discrete codebook, $\mathcal{C}=\left\{\mathbf{u L}, \mathbf{u} \in \mathbb{Z}_{p}^{k}\right\}$

2) Construction of the lattice $\Lambda^{*}$ by dividing all the elements of $\mathcal{C}$ by $p$ and replicating over $\mathbb{Z}^{n}, \Lambda^{*}=p^{-1} \mathcal{C}+\mathbb{Z}^{n}$

3) Construction of the Fine lattice by rotating $\Lambda^{*}$ by the generator matrix of the coarse lattice $\Lambda_{\mathrm{F}}=\mathcal{C}+p \mathbb{Z}^{n}$

We consider in this work a simple coding scheme by taking $p=11, k=1$ and $n=2$ and the linear code $C$ whose generator matrix is $\mathbf{L}=\left[\begin{array}{ll}2 & 3\end{array}\right]$. The coarse lattice in our case is $\Lambda_{\mathrm{C}}=11 \mathbb{Z}^{2}$ and the Fine lattice is $\Lambda_{\mathrm{F}}=\mathcal{C}+11 \mathbb{Z}^{2}$. In Fig.3 we plot a portion of the Fine lattice as well as the code $\mathcal{C}$.

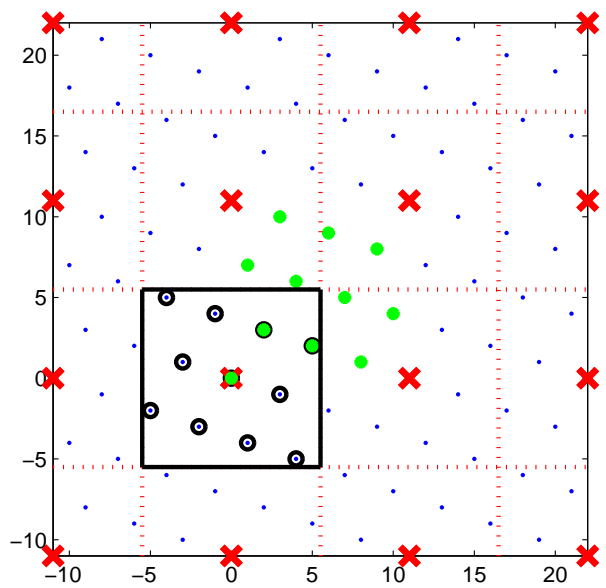

Fig. 3. Nested lattice Code: small blue points are points of the Fine lattice, cross points are coarse lattice points. Dashed lines delimit the voronoi regions of the coarse lattice. Bold square is the fundamental voronoi region of $\Lambda_{\mathrm{C}}$ Points of the Fine lattice inside this region form the nested lattice code $\mathcal{C}$.

\section{B. Processing steps at the receiver}

Processing steps at the IF receiver are the following:

i) Given $\mathbf{H}$, select optimal coefficient matrix $\mathbf{A}_{\mathrm{opt}}$ according to described algorithms in section III.

ii) Compute $\mathbf{B}_{\mathrm{opt}}=\mathbf{A}_{\mathrm{opt}} \mathbf{H}^{t}\left(\mathbf{H} \mathbf{H}^{t}+\frac{1}{\rho} \mathbf{I}\right)^{-1}$ and scale the channel output.

iii) Perform minimum distance decoding to estimate from $\hat{\mathbf{y}}_{m}, \hat{\lambda}_{m}=\operatorname{argmin}_{\lambda \in \Lambda_{\mathrm{F}}}\left\|\hat{\mathbf{y}}_{m}-\lambda\right\|^{2}$. The Sphere Decoder can be used to accomplish this step.

iv) Solve the linear system $\Lambda=\mathbf{A}_{\text {opt }} \mathbf{X}$ to estimate codewords $\hat{\mathbf{x}}_{1}, \ldots, \hat{\mathbf{x}}_{M}$.

v) Map estimated codewords to finite field. This is done as follows: first perform the modulo operation with respect to the coarse lattice in order to garantee that the codeword 
belongs to the nested lattice code, then map the resulting codeword to finite field using the inverse mapping $\Phi^{-1}$. Then for $\hat{\mathbf{w}}_{i}=\Phi^{-1}\left(\left[\hat{\mathbf{x}}_{i}\right] \bmod \Lambda_{\mathrm{C}}\right), i=1, \ldots, M$.

\section{Performance Evaluation in a $2 \times 2$ MiMO System}

We address in this section performance evaluation of the proposed coding scheme in a $2 \times 2$ MIMO system. Monte-carlo simulations have been carried out to evaluate both average achievable rates and codeword error probability at the receiver for the different studied receivers: linear receivers through the ' $\mathrm{ZF}$ ' and the 'MMSE', lattice-reduction aided linear receivers through the 'LLL+ZF' and 'LLL+MMSE' as well as the proposed implementation for the Integer Forcing Linear receivers ('IF'). Performance of these receivers is compared to the optimal joint ML decoder.

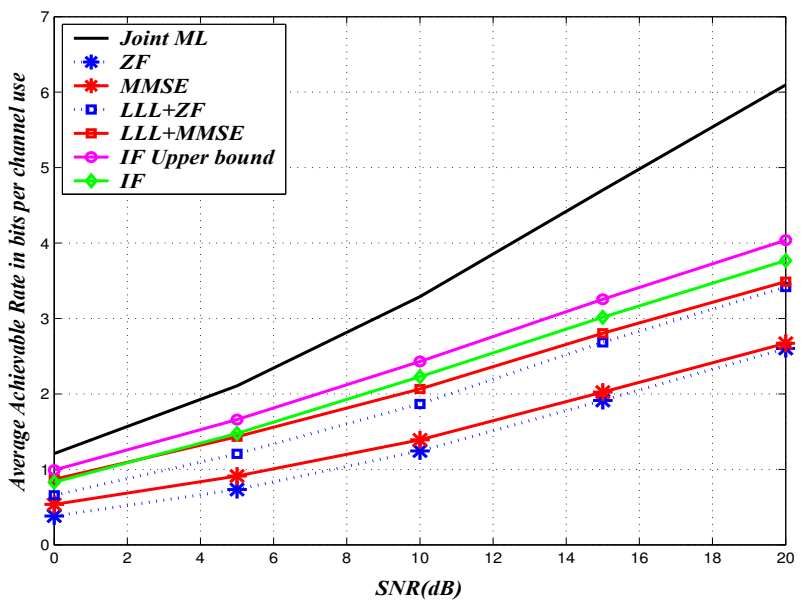

Fig. 4. Average Achievable Rate for $2 \times 2$ MIMO system.

Starting with the achievable rates plotted in Fig.4, we first point out that LR-aided linear receivers perform better than the linear receivers. A gain of $5-\mathrm{dB}$ of the 'LLL+ZF' over the ' $\mathrm{ZF}$ ' is reported for a target rate of 2 bits/channel use. In addition, numerical results confirm that the Integer Forcing receiver outperforms the 'ZF' and 'MMSE' and even LatticeReduction aided linear receivers. The proposed algorithm allows to achieve a gain of $1.4 \mathrm{~dB}$ over the 'LLL+MMSE' and $2 \mathrm{~dB}$ over the 'LLL+ZF' for a target rate of 3 bits/channel use. This result confirms the suboptimality of restricting the equivalent channel matrix $\mathbf{A}$ in lattice reduction aided receivers to be unimodular. Furthermore, the proposed algorithm allows to approach the upper bound of the Integer Forcing receiver given by $\frac{1}{2} \log \left(1+\rho \sigma_{\max }^{2}\right)$, and reduces the loss to $1.4 \mathrm{~dB}$. However, compared to the ML decoder whose achievable rate is given by [1] $R_{\text {Joint }}=\frac{1}{2} \log \left(\mathbf{I}_{N}+\rho \mathbf{H} \mathbf{H}^{t}\right)$, the proposed architecture presents a considerable gap to the joint ML that overtakes $6 \mathrm{~dB}$ for SNR values greater than $16 \mathrm{~dB}$.

Now as far as the error probability is concerned, same deduction can be made as illustrated in Fig.5: Integer Forcing Linear receivers outperform both linear receivers and LR-aided linear receivers. For a codeword error rate equal to $10^{-2}$ the gain of the 'IF' over the 'LLL+ZF' and 'LLL+MMSE' is $5 \mathrm{~dB}$ and about $17 \mathrm{~dB}$ over the ' $\mathrm{ZF}$ '. The gap between the proposed 'IF' algorithm to the ML joint decoder counts $5 \mathrm{~dB}$.

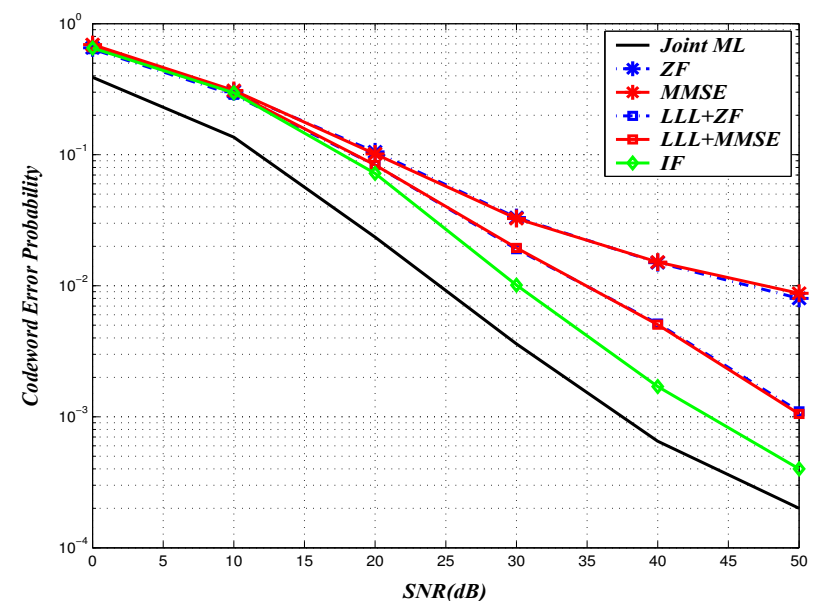

Fig. 5. Codeword Error probability for $2 \times 2$ MIMO system.

\section{CONCLUSION}

In this work we proposed an implementation of new promising integer forcing linear receivers for MIMO channels: we developed algorithms to solve for optimal receiver parameters and address error performance evaluation in practical scenarios where low-complexity lattice codes are used. Numerical results are a proof of concept on the outperformance of this new architecture over linear receivers and LR-aided linear receivers. In order to make practical wireless receivers take advantage of the promised gains of this new design, the decoding complexity needs to be investigated.

\section{ACKNOWLEDGMENTS}

The authors would like to acknowledge the European Commission for funding SmartEN ITN (Grant No. 238726) under the Marie Curie ITN FP7 program.

\section{REFERENCES}

[1] Z. Jiening, U. Erez, M. Gastpar, and B. Nazer. Mimo compute-andforward. In Proceedings of ISIT, pages 2848 -2852, july 2009.

[2] Z. Jiening, B. Nazer, U. Erez, and M. Gastpar. Integer-forcing linear receivers: A new low-complexity mimo architecture. In Proceedings of Vehicular Technology Conference Fall, pages 1 -5, sept. 2010.

[3] Z. Jiening, B. Nazer, U. Erez, and M. Gastpar. Integer-forcing linear receivers. In Proceedings of ISIT, pages 1022 -1026, june 2010.

[4] Jiening Zhan, U. Erez, M. Gastpar, and B. Nazer. Mitigating interference with integer-forcing architectures. In Proceedings of ISIT, pages 1673 -1677 , aug. 2011.

[5] A. K. Lenstra, H. W. Lenstra, and L. Lovász. Factoring polynomials with rational coefficients. Math. Ann., 261:515-534, 1982.

[6] U. Fincke and M. Pohst. Improved Methods for Calculating Vectors of Short Length in a Lattice, Including a Complexity Analysis. Mathematics of Computation, 44(170):463-471, 1985.

[7] U. Erez, S. Litsyn, and R. Zamir. Lattices which are good for (almost) everything. In Proceedings of ITW, pages $271-274$, april 2003.

[8] B. Nazer and M. Gastpar. Compute-and-forward: Harnessing interference with structured codes. In Proceedings of ISIT, pages 772 -776, july 2008

[9] J. Conway and N.J.A. Sloan. Sphere Packings, Lattices and Groups, volume 290 of Grundlehren der mathematischen Wissenschaften. Springer, 3rd edition, 1998. 